\title{
Iliac artery pseudoaneurysm presenting as a paravertebral collection: a potentially fatal mimic
}

\author{
Sawsan Taif, ${ }^{1}$ Asif Alrawi, ${ }^{2}$ Jokha Al-Kalbani ${ }^{3}$
}

${ }^{1}$ Department of Radiology, Khoula Hospital, Muscat, Oman

${ }^{2}$ Department of Orthopedics, Khoula Hospital, Muscat, Oman

${ }^{3}$ Department of Radiology, Royal Hospital, Muscat, Oman

\section{Correspondence to} Dr Sawsan Taif,

sawsantaif@gmail.com

Accepted 24 February 2014

\section{SUMMARY}

Pseudoaneurysms of the iliac arteries are extremely rare and can complicate trauma, surgical or interventional procedures. We report a case of pseudoaneurysm arising from the common iliac artery in a 37-year-old man which presented as a paravertebral collection. As the MRI appearance mimicked spondylodiscitis, a CT guided biopsy was requested. However, the presence of a signal void centre guided the radiologist to the correct diagnosis, which was later confirmed on contrastenhanced CT. Following unsuccessful treatment by percutaneous thrombin injection, open surgery was performed which revealed a leaking aneurysm associated with a huge retroperitoneal haematoma. We present this case to highlight the importance of considering vascular lesions as a differential diagnosis in patients presenting with a mass or pressure symptoms in different parts of the body. Misdiagnosing such lesions as soft tissue tumours or abscesses may result in serious consequences.

\section{BACKGROUND}

Aneurysms and other vascular lesions can involve different arteries in the body and therefore may only be discovered during routine investigation of non-specific symptoms such as pain. Failure to identify such lesions on imaging studies may result in attempted intervention with catastrophic events. We present this case of an iliac artery pseudoaneurysm presenting as a paravertebral collection to draw attention to such scenarios and highlight the importance of considering the possibility of a vascular lesion.

\section{CASE PRESENTATION}

A 37-year-old man presented to the orthopaedic department with 2-month history of back pain. The pain was severe, interfered with the patient's activity, and occasionally radiated to both lower limbs. He had no bladder or bowel dysfunction or other systemic manifestations. On examination, the patient had a decreased range of back movements due to pain but had no neurological deficits. A vague mass was felt in the lower aspect on the right side of abdomen. The lumbar spine radiograph and initial blood investigations were unremarkable. Lumbar spine MRI was performed and showed a destructive lesion in the L4 vertebral body, associated with a large thick-walled paravertebral structure showing variable signal intensities (figure 1). This lesion was located on the right side, medial to the psoas muscle and adjacent to the L4-5 intervertebral disc. In light of these imaging findings, a differential diagnosis was made which included spondylodiscitis with collection and a paravertebral tumour. As a result, the patient was referred to the radiology department for CT guided biopsy. However, before performing the biopsy, the radiologist reviewed the MRI images and noticed that the paravertebral lesion showed a signal void centre and variable hyperintense peripheral signals. Consequently, a contrast-enhanced CT scan for further evaluation of the lesion revealed a large irregular-shaped pseudoaneurysm measuring $8.5 \times 7 \times 6 \mathrm{~cm}$ and arising from the site of the bifurcation of the right common iliac artery (figure 2). It extended superiorly and posteriorly to the prevertebral space and caused erosion of the L4 vertebral body. The wall of the aneurysm demonstrated peripheral hypodense non-contrast filled areas on CT, denoting a thrombus. Furthermore, the pseudoaneurysm was compressing the right common femoral vein which showed sluggish flow on Doppler studies. The cause of the pseudoaneurysm was not clear, but the patient gave a history of bladder surgery 20 years earlier.

\section{DIFFERENTIAL DIAGNOSIS}

This type of pseudoaneurysm manifesting as a paravertebral lesion can mimic spondylodiscitis with fluid collections due to pyogenic or tuberculous pathology. Paravertebral masses such as lymphoma, neurogenic tumours and metastases are other important differential diagnoses.

\section{TREATMENT}

The patient was referred to the vascular surgeon who decided to use minimally invasive techniques. Percutaneous thrombin injection under US guidance was initially tried but failed to effectively control the large aneurysm. In addition, the patient reported pain and oedema of the right lower limb few hours after the procedure. A Doppler study showed weak signals in the right lower limb arteries, which raised the suspicion of arterial thrombosis complicating thrombin injection. Subsequently, CT angiography confirmed the residual aneurysm as well as an embolus in the right common femoral artery. Increasing leakage from this pseudoaneurysm had also resulted in the formation of a large new retroperitoneal haematoma that reached up to the right kidney which was pushed anteriorly (figure 3 ). The patient also began to have mild coagulation dysfunction. At this stage, 

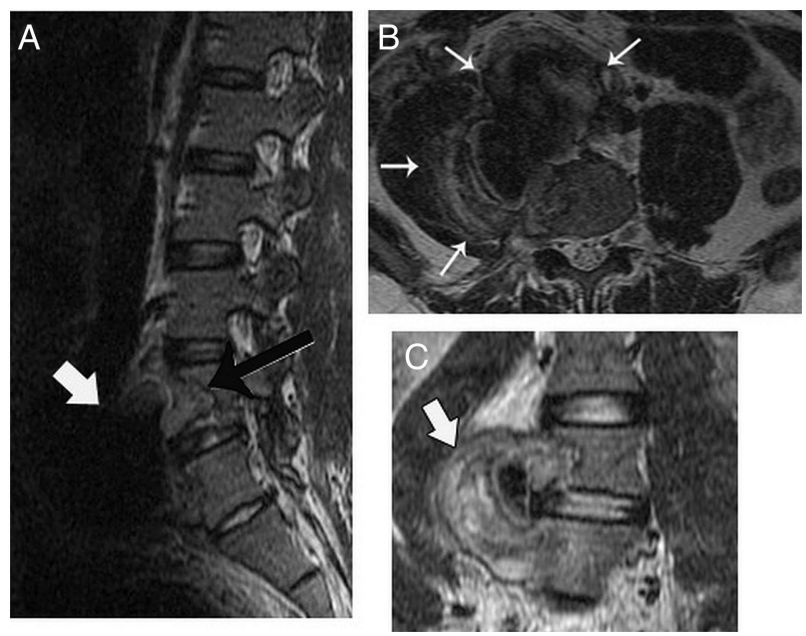

Figure 1 T2-weighted MRI in three planes: (A) sagittal, (B) axial and (C) coronal. Images show a bony lesion involving the anterior aspect of the $L 4$ vertebral body adjacent to the $L 4-5$ disc (black arrow). There is a right paravertebral structure (white arrows) with a signal void centre and variable, predominantly hyperintense, peripheral signals (white arrows) simulating collection.

the vascular surgeon decided to perform urgent open surgery to treat the residual aneurysm and remove the femoral artery embolus. Intraoperatively, the retroperitoneum was approached with exposure of all involved vessels. A large common iliac artery leaking aneurysm was found, indicating a defect in the posterior wall. Aneurysmectomy and evacuation of the large retroperitoneal haematoma were performed. The surgeon then
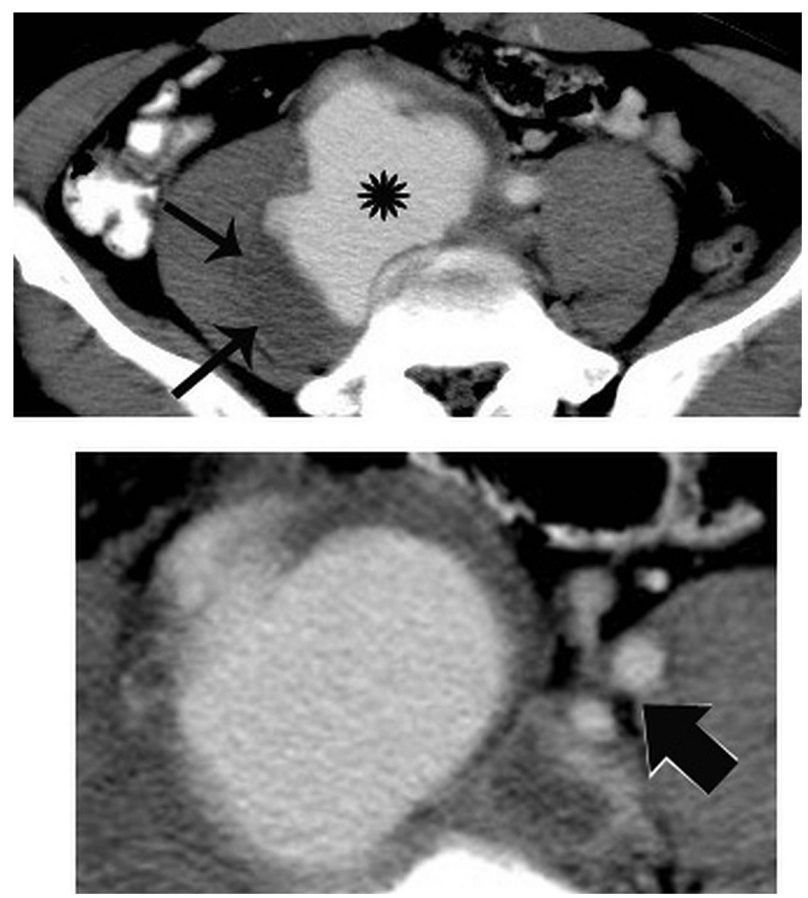

Figure 2 Contrast-enhanced CT scan of the abdomen and pelvis, with axial images showing a large contrast-filled structure in keeping with iliac artery pseudoaneurysm (asterisk). The left internal and external iliac arteries (thick black arrow) and the surrounding hypodense thrombus (thin black arrows) are also noted. carried out an iliofemoral bypass using a polytetrafluoroethylene (PTFE) graft. The right femoral artery embolus was also removed through an additional groin incision.

\section{OUTCOME AND FOLLOW-UP}

Postoperative CT angiography revealed effective occlusion of the pseudoaneurysm and patent common iliac and common femoral arteries (figure 4). The patient's condition gradually improved with general supportive measures. Three weeks after surgery, he was discharged with no recurrence of the aneurysm at 3-year follow-up.

\section{DISCUSSION}

Isolated true and false iliac artery aneurysms are rare. Cases of iliac artery pseudoaneurysms are even less common and very rarely reported in the literature.

A pseudoaneurysm is a contained rupture of an artery due to disruption of the wall continuity. It forms a sac in direct communication with the donor artery. Unlike a true aneurysm which shows all three wall layers, the pseudoaneurysm is surrounded by only a thin medial or adventitial layer.

Many pseudoaneurysms are caused by blunt or penetrating trauma. Recently, the number of iatrogenic pseudoaneurysms due to surgical and interventional procedures has increased. Infection is a cause of both pseudoaneurysms and true aneurysms; however, pseudoaneurysms are more frequent due to easy disruption of the arterial wall. Connective tissue disorders, vasculitis, inflammation and erosion secondary to malignancy are other reported causes of pseudoaneurysms. ${ }^{1-3}$ Although atherosclerotic aneurysms are usually true, pseudoaneurysms originating from penetrating atherosclerotic ulcers have also been described.

Patients can present with a variety of symptoms based on the location of the aneurysm. Rupture is common in patients with iliac artery pseudoaneurysms and is associated with a high mortality rate approaching 50\%. Presentation with pressure effects on the surrounding structures or rectal bleeding due to fistula formation has also been reported. ${ }^{4}$

On MRI, aneurysms demonstrate a signal void centre representing the aneurysm lumen, with variable heterogeneous peripheral signals representing the thrombus/haematoma. In comparison to other types of imaging, these changes are difficult to recognise and interpret, making MRI diagnosis more challenging. However, aneurysms may be first discovered on MRI performed for non-specific symptoms such as pain or pressure effects. As a result, radiologists should be able to recognise vascular lesions and to differentiate their appearance from other more common soft tissue lesions. An important clue to the diagnosis of aneurysms is the presence of a signal void centre on all MRI sequences, which should alert the radiologist to the possibility of a vascular lesion.

When an aneurysm is suspected, Doppler ultrasonography is a valuable initial tool that can allow rapid diagnosis. It has the advantage of being an non-invasive, readily available and nonexpensive bedside investigation. It shows the aneurysm as a hypoechoic fluid-containing structure which demonstrates blood flow and is located adjacent to the supplying artery.

With the advent of multidetector CT scanners, contrast-enhanced CT is considered the gold standard test with a high sensitivity and specificity for arterial injury. Catheter angiography, which was previously considered the best diagnostic tool, has now largely been replaced by CT. The pseudoaneurysm is shown as a thin-walled, irregular-shaped and contrast-filled sac in direct communication with the donor artery. CT also has 
Figure 3 Evidence of failure of percutaneous thrombin injection is seen on CT angiography. Multiplanar reformatted images show the residual smaller aneurysm (white arrows). The axial image shows a retroperitoneal haematoma pushing the kidney anteriorly (black arrows). A hypodense embolus is seen in the right femoral artery (asterisk).
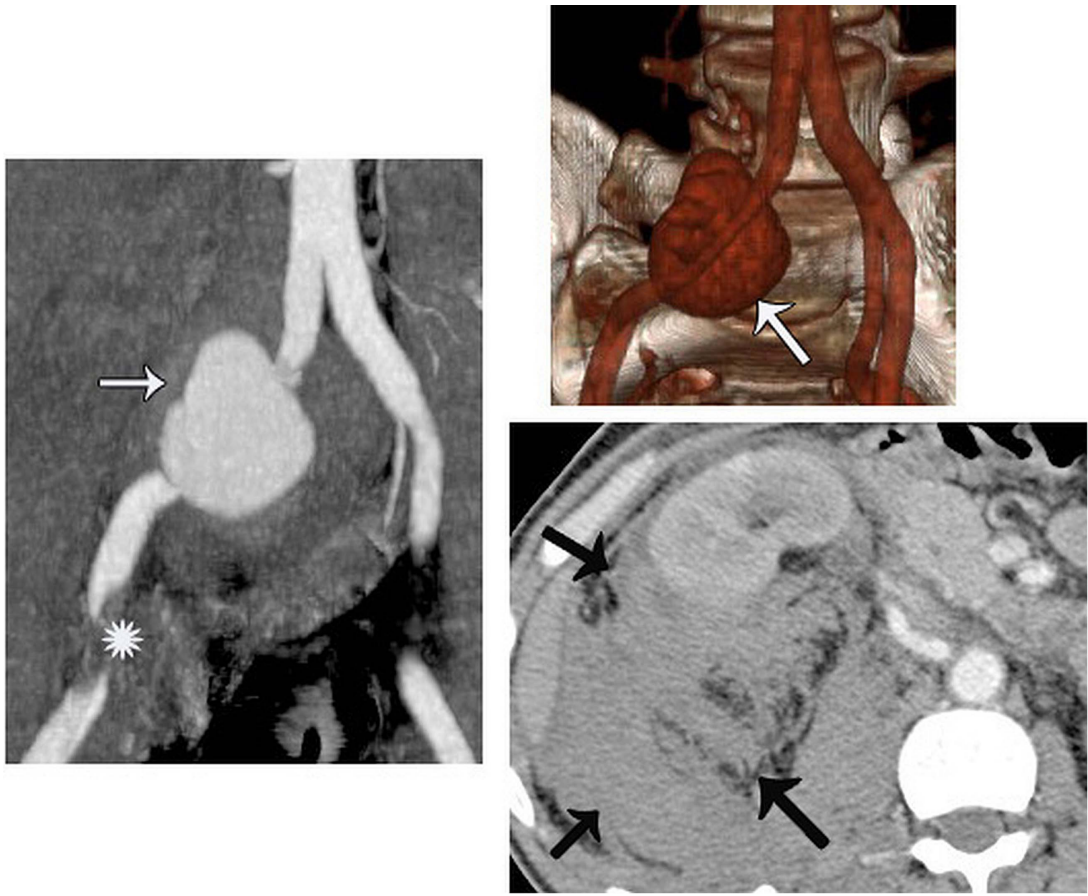

the advantage of allowing multiplanar reconstruction to create high resolution reformatted images in two and three dimensional planes. ${ }^{5}$

Once the diagnosis has been established, patients should be referred to the vascular specialist for management. Symptomatic pseudoaneurysms showing intermittent or continuous bleeding, or rapid expansion, and asymptomatic large aneurysms must be treated. The management of asymptomatic small pseudoaneurysms is controversial. Although these lesions can undergo spontaneous thrombosis, most authors argue against conservative management as the natural history of these lesions is unpredictable. Life-threatening spontaneous rupture may occur at any time, with the outcome depending on the anatomical location and clinical setting. ${ }^{3}$ Therefore, many authors believe that definitive treatment must be provided in most cases.
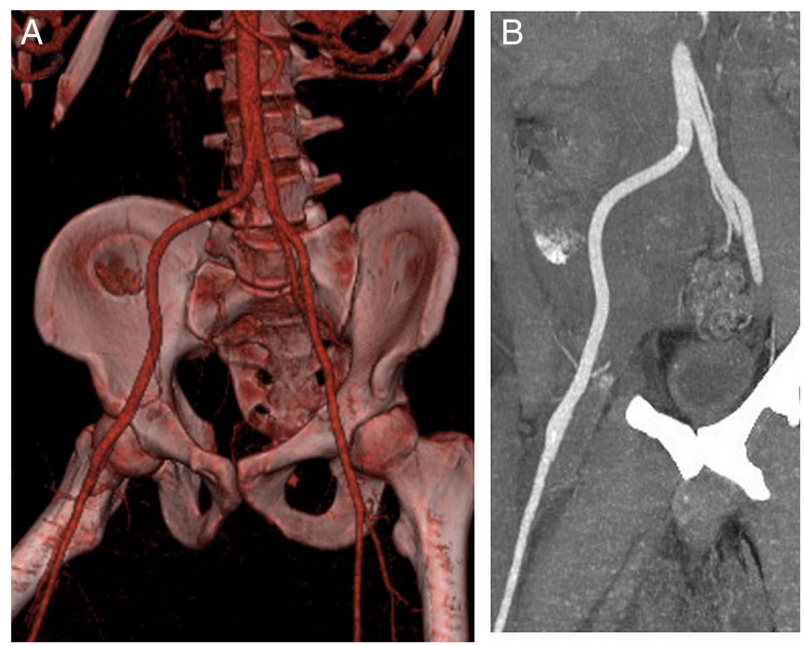

Figure 4 Postoperative $\mathrm{CT}$ angiography. (A) Volume-rendered reformat and (B) maximum intensity projection images showing a successfully treated right common iliac artery pseudoaneurysm with normally appearing arteries.
Open surgery is the traditional method for treating pseudoaneurysms and is still frequently employed. However, minimally invasive procedures such as percutaneous thrombin injection and endovascular treatment by embolisation and stent-graft are increasingly used for the treatment of these lesions. ${ }^{2} 3$ US-guided compression, previously used in the management of some cases, has now generally been replaced by other more effective and practical techniques.

The aim of percutaneous thrombin injection is to induce aneurysm thrombosis, while endovascular treatment serves to exclude the pseudoaneurysm from the circulation and systemic blood pressure, thereby preventing further expansion. The choice of treatment depends on the location of the pseudoaneurysm, the surrounding structures, the vascular anatomy and patient comorbidities.

The literature has shown that the use of non-invasive procedures for the treatment of pseudoaneurysms has led to a reduction in patient morbidity and mortality. ${ }^{3}{ }^{6-8}$ However, in the current case, percutaneous thrombin injection was complicated by thrombosis of the femoral artery. Complication rates as low as $4 \%$ have been reported in the literature following this procedure. Thromboembolic events arise from thrombin flow beyond site of the aneurysm into the arterial system. ${ }^{3}$ Some studies have shown this method is more successful in acute pseudoaneurysms within the first few weeks of their development. ${ }^{9}$ Many authors also consider this method unsuitable for treating aneurysms with a wide neck as they have a slightly higher risk of embolisation.

Despite the emerging role of endovascular treatment, surgery is still indicated in many cases. This is the method of choice in the presence of local mass effects and in cases where minimally invasive treatment has failed. Many authors recommend surgical treatment in patients with infected pseudoaneurysms. However, the presence of infection is not an absolute contraindication to minimally invasive techniques, and management should be tailored to the individual case. Careful evaluation of the imaging studies, particularly CT angiography, is essential to determine the proper treatment and hence reduce the risk of complications in order to achieve the best possible results. ${ }^{1-39}$ 


\section{Learning points}

- Aneurysms can masquerade as a mass or an abscess, so careful evaluation of imaging studies is essential to rule out a vascular origin prior to lesion intervention.

- Vascular lesions may be discovered incidentally during routine investigation of non-specific symptoms such as pain in a particular location, and so radiologists must be familiar with their imaging findings, particularly on MRI.

- Contrast-enhanced CT is the gold standard for the investigation of lesions suspected of having a vascular origin.

- Although minimally invasive procedures, such as percutaneous thrombin injection, are considered to be safe in comparison to open surgery, they may still result in serious complications, so careful evaluation is required to determine optimal treatment.

Contributors The case report was written by ST with the help and support of AA and JA-K.
Competing interests None.

Patient consent Obtained.

Provenance and peer review Not commissioned; externally peer reviewed.

\section{REFERENCES}

1 Sueyoshi E, Sakamoto I, Nakashima K, et al. Visceral and peripheral arterial pseudoaneurysms. AJR Am J Roentgenol 2005;185:741-9.

2 Ferreira J, Canedo A, Brandão D, et al. Isolated iliac artery aneurysms: six-year experience. Interact Cardiovasc Thorac Surg 2010;10:245-8.

3 Saad NE, Saad WE, Davies MG, et al. Pseudoaneurysms and the role of minimally invasive techniques in their management. Radiographics 2005;25:S173-89.

4 Arthur TI, Gillespie CJ, Butcher W. Pseudoaneurysm of the internal iliac artery resulting in massive per-rectal bleeding. JSCR 2013;10.

5 Soto JA, Munera F, Morales C, et al. Focal arterial injuries of the proximal extremities: helical CT arteriography as the initial method of diagnosis. Radiology 2001;218:188-94.

6 Chaer RA, Barbato JE, Lin CS, et al. Isolated iliac artery aneurysms: a contemporary comparison of endovascular and open repair. J Vasc Surg 2008;47:708-13.

7 Bacharach JM, Slovut D. State of the art: management of iliac artery aneurysmal disease. Catheter Cardiovasc Interv 2008;71:708-14.

8 Pitoulias GA, Donas KP, Schulte $S$, et al. Isolated iliac artery aneurysms: endovascular versus open elective repair. J Vasc Surg 2007;46:648-54.

9 Gurel K, Gur S, Ozkan U, et al. US-guided percutaneous thrombin injection of postcatheterization pseudoaneurysms. Diagn Interv Radiol 2012;18:319-25.

Copyright 2014 BMJ Publishing Group. All rights reserved. For permission to reuse any of this content visit http://group.bmj.com/group/rights-licensing/permissions.

BMJ Case Report Fellows may re-use this article for personal use and teaching without any further permission.

Become a Fellow of BMJ Case Reports today and you can:

- Submit as many cases as you like

- Enjoy fast sympathetic peer review and rapid publication of accepted articles

- Access all the published articles

- Re-use any of the published material for personal use and teaching without further permission

For information on Institutional Fellowships contact consortiasales@bmjgroup.com

Visit casereports.bmj.com for more articles like this and to become a Fellow 